Du Directoire au Consulat. 1. Le lien politique local dans la grande nation - Du Directoire au Consulat. 2. L'intégration des citoyens dans la grande nation

Annie Duprat et Raymonde Monnier

(2) OpenEdition

Journals

Édition électronique

URL : https://journals.openedition.org/ahrf/1117

DOI : 10.4000/ahrf.1117

ISSN : 1952-403X

Éditeur :

Armand Colin, Société des études robespierristes

Édition imprimée

Date de publication : 1 juin 2001

Pagination : 206-208

ISSN : 0003-4436

Référence électronique

Annie Duprat et Raymonde Monnier, « Du Directoire au Consulat. 1. Le lien politique local dans la grande nation - Du Directoire au Consulat. 2. L'intégration des citoyens dans la grande nation », Annales historiques de la Révolution française [En ligne], 324 | avril-juin 2001, mis en ligne le 10 avril 2006, consulté le 24 avril 2022. URL : http://journals.openedition.org/ahrf/1117 ; DOI : https://doi.org/ 10.4000/ahrf.1117

Ce document a été généré automatiquement le 24 avril 2022.

Tous droits réservés 


\title{
Du Directoire au Consulat. 1. Le lien politique local dans la grande nation - Du Directoire au Consulat. 2. L'intégration des citoyens dans la grande nation
}

\author{
Annie Duprat et Raymonde Monnier
}

\section{RÉFÉRENCE}

Du Directoire au Consulat. 1. Le lien politique local dans la grande nation, Jacques Bernet, Jean-Pierre Jessenne, Hervé Leuwers éd., Centre de Recherche sur l'Histoire de l'Europe du Nord-Ouest, vol. 20, Villeneuve d'Ascq, ANRT, Lille 3, 1999, 336 p. 170 F. Du Directoire au Consulat. 2. L'intégration des citoyens dans la grande nation, Hervé Leuwers éd. avec la collaboration de Jacques Bernet et Jean-Pierre Jessenne, Centre de Recherche sur l'Histoire de l'Europe du Nord-Ouest, vol. 22, Villeneuve d'Ascq, ANRT, Lille 3, 2000, 317 p. $170 \mathrm{~F}$.

1 Des liens étroits existent entre ces deux volumes qui publient les actes de tables rondes, tenues en 1998 et 1999 dans le cadre du programme de rencontres sur le Directoire et le Consulat, organisées conjointement par le CHRIV de cette université, le CHHEN-O (Lille 3), et l'IRED (Rouen), à l'initiative de Jean-Pierre Jessenne. La première table ronde, tenue à l'Université de Valenciennes et du Hainaut-Cambrésis en mars 1998, à laquelle ont activement contribué des chercheurs de l'Université catholique de Louvain et de l'Université libre de Bruxelles, a réuni une quarantaine de participants français, belges et allemands. Il s'agissait, à la suite du colloque international de Clermont-Ferrand sur la République directoriale (voir le compte rendu, AHRF, $\mathrm{n}^{\circ} 314,1998$, pp. 778-780), de réévaluer l'histoire de cette période de transition, cette fois plus précisément à la lumière des travaux récents sur le pouvoir et les institutions locales, autour de la 
notion du «lien social». Après les rapports introductifs, qui résument l'état des questions (C. Bruneel, B. Gainot, J.-P. Jessenne), une première partie est consacrée aux dynamiques locales d'engagement ou de refus politique des citoyens vis-à-vis d'un régime qui se voulait celui du juste milieu, entre le retour à l'Ancien Régime et les excès de la terreur; la seconde partie traite de la greffe locale, plus ou moins réussie, de quelques institutions du Directoire.

2 Ces études régionales, menées dans une optique comparative, ne sont pas bornées aux limites de l'ancienne France, des provinces globalement conformistes du Nord ou du Bassin parisien, à celles d'anti- ou de Contre-Révolution, comme l'Ouest ou le Midi, mais s'étendent aux territoires réunis, avec l'exemple de la Belgique (L. Dhont). Elles permettent de nuancer une typologie des engagements politiques, opposant sous le Directoire une France méridionale secouée de troubles à celle du Nord-Ouest, plus consensuelle. Or le fractionnement de l'autorité et du système territorial de l'an IV à l'an VIII, qui constitue une des faiblesses majeures du régime, s'avère avoir ici un effet paradoxal quant à l'intérêt porté aux affaires publiques, qui ne se traduit pas par une apathie générale mais par de brusques poussées de participation électorale dans les villes. L'exemple de la Provence ou d'Avignon, avec les affrontements violents de «l'affaire» de pluviôse an V (C. Peyrard), comme ceux de l'Ouest ou de la BasseNormandie (R. Dupuy, S. Denys-Blondeau), montrent le durcissement des clivages politiques, et des engagements contrastés dans les zones sporadiquement troublées par la violence «blanche». Ailleurs se lit chez les notables une volonté affirmée de stabilisation politique dans un esprit de compromis, ainsi en Belgique (malgré la Guerre dite des paysans en 1798) ou en Auvergne, grâce aux pratiques culturelles et aux réseaux républicains qui structurent la vie politique locale (P. Bourdin); ainsi en va-t-il également des fêtes civiques liégeoises (P. Raxhon). La sociabilité joue un rôle non négligeable dans le ralliement au régime, notamment celle des loges maçonniques en plein réveil; bien qu'en marge de la sociabilité officielle, elles redeviennent un enjeu dans un champ politique, social et culturel en plein renouvellement (P.-Y. Beaurepaire). Le compromis est plus difficile en matière religieuse, où la Révolution a engendré une situation confuse. Le Concordat favorise la stabilisation en Belgique, en apportant l'apaisement sur un point qui était un ferment permanent d'opposition.

Du côté des institutions, comment fut acceptée, après une gestation laborieuse ( $M$. Pertué), la mise en place et l'expérience innovante et éphémère des municipalités cantonales? Des études ponctuelles dans deux régions globalement attachées à la République, l'île-de-France (S. Bianchi) et la Picardie (J. Bernet) montrent que le bilan doit être nuancé. Idée minoritaire mais constante dans la réflexion politique depuis le début de la Révolution, les municipalités cantonales eurent leurs partisans tout au long du xixe siècle. L'expérience, qui rencontra des difficultés faute de moyens, répondit finalement aux attentes du Directoire en recentrant les activités des administrateurs locaux sur les problèmes des contributions, des subsistances et du ravitaillement; en évitant les troubles tout en satisfaisant aux besoins de la République et des armées, elles ont fait preuve d'une réelle efficacité en leur temps. Le déplacement de la vie politique au profit du chef-lieu de canton correspondait aux logiques profondes de l'évolution d'un personnel de notables et des relations entre le pouvoir local et l'État. L'introduction du modèle français dans les villes belges des Deux-Nèthes, Anvers, Malines, Lierre et Turnhout (F. Stevens), n'alla pas sans difficultés dans un département où les élections sont annulées en fructidor an $\mathrm{V}$, et où l'année suivante la loi sur la 
conscription suscite l'insurrection à Malines. Des différentes évolutions locales se dégage une situation chaotique, où les représentants doivent faire face au mauvais gré des populations et des officiers municipaux, dans une région de tendance conservatrice où seule l'élite maîtrise la langue française. Déjà bien expérimentées en France, mais nouvellement implantées en Belgique, les institutions judiciaires ont contribué à l'encadrement et à l'acculturation des citoyens dans le Nord et les départements belges (X. Rousseaux). En dépit de multiples difficultés, la justice criminelle remplit sa mission avec constance dans le département du Nord (D. Moyaux). Les justices de paix quant à elles correspondaient à cette justice de proximité très prisée des citoyens, comme le montre l'exemple des départements du Nord (S. Humbert-Convain) et de la Dyle (J. Logie), et de districts du Pas-de-Calais et de Seine-Inférieure (P. Bréemersch, K. Conseil). En Belgique, après les difficultés d'installation de la justice et du code pénal, l'Empire permit une acculturation relativement durable au système judiciaire de la Révolution française. Sans doute conviendrait-il de multiplier ces études de terrain et d'étendre ce tour d'horizon de la greffe des institutions, pour apprécier l'œuvre du Directoire et le redéploiement des pouvoirs, tandis que s'affirme la "grande nation» (J.Y. Guiomar), une expression apparue à l'été 1797 et dont saura tirer profit le général Bonaparte.

4 Les travaux entrepris à Valenciennes se sont poursuivis en mars 1999, à Lille, avec une vingtaine de chercheurs placés sous la même direction scientifique (Jacques Bernet, Jean-Pierre Jessenne et Hervé Leuwers). La première partie du volume, qui réunit neuf contributions porte spécifiquement sur la définition (ou plutôt sur «les» définitions, plurielles ou successives) de la notion de «Grande Nation», naguère étudiée par Jacques Godechot, à la suite des réflexions de Jean-Yves Guiomar dans son rapport introductif à la table ronde de Valenciennes. L'ensemble du volume s'inscrit dans une démarche d'histoire historicisante qui ne néglige cependant pas les perspectives conceptuelles dont la nécessité apparaît de plus en plus clairement dans le grand chantier des études révolutionnaires. Trois directions ont été empruntées par les auteurs: le débat théorique sur l'historiographie du discours théorique sur la «Grande Nation» et sa réception, contemporaine et immédiatement postérieure. Tandis que J-C. Martin reprend le dossier de façon théorique, en le replaçant dans les débats de grande envergure touchant à la philosophie politique, avec une méthode précise portant essentiellement sur une relecture du Moniteur. J-Y. Guiomar s'attache à présenter des études de cas et des directions de recherche. B. Gainot confronte l'usage des termes («Grande Nation») au miroir de leur utilisation, au cours de la séquence 1797-1799, dans les adresses envoyées dans les départements du nord de cette «France étendue»; d'autres auteurs enrichissent le dossier à partir d'exemples spécifiques régionaux en France (P. Bourdin) mais également dans les régions qui lui sont un temps rattachées ou soumises à son influence, la Belgique, les Pays-Bas, la Rhénanie, la Suisse ( $\mathrm{H}$. Leuwers, M. Gilli, A. Jourdan, A-J. Czouz-Tornare); enfin, loin des exhortations patriotiques, A. Crépin a démontré comment les réactions de défense des communautés villageoises face à la conscription révélaient des solidarités villageoises transfrontalières dès lors que les administrateurs locaux n'avaient pas su (ou pas pu?) emporter l'adhésion des populations.

5 La seconde partie s'attache à mettre en évidence quels sont les facteurs d'intégration sociale à l'époque directoriale, des questions religieuses aux questions économiques. L'évolution des pratiques religieuses, la réorganisation de l'Église de France après le rétablissement de la liberté des cultes par la Convention thermidorienne en 1795, les 
relations de cette église gallicane avec la papauté, avant et pendant le grand Concile national de 1797, ainsi que la condition matérielle, plus que misérable, des desservants, montrent que se joue là une partition de dissociation sociale davantage que d'unité. Placées à différentes échelles, de la synthèse nationale (B. Plongeron) aux études locales, sur la Picardie (J. Bernet) et sur la Rhénanie (W-H. Stein), ces trois études particulièrement précises sont d'autant plus importantes qu'elles entreprennent de nourrir un champ de recherches quelque peu délaissé ces dernières années, comme le signale G. Deregnaucourt. Enfin, sous le titre «les dimensions économique, financière, sociale et culturelle de la Grande Nation», P. Guignet introduit les six dernières communications, tout en montrant combien les études portant sur la politique d'enseignement (S. Bianchi), d'assistance publique et hospitalière (Y. Marec et L. Delporte) auraient pu s'inscrire dans le champ du religieux. L'article très documenté de S. Bianchi apporte des informations indispensables pour reprendre le dossier de l'enseignement primaire sous l'angle du lien social, à partir des correspondances de François de Neuchâteau avec les commissaires des départements et des enquêtes entreprises en l'an VI et en l'an IX. Enfin, les travaux précis sur les administrations dédiées aux finances, en France et en Belgique (M. de Oliveira) ou les réformes de l'État directorial en matière de fiscalité (c. Wolikow), qui conduisent à dessaisir les communes de l'établissement des rôles de l'impôt, montrent à nouveau, et parfois a contrario, l'émergence de nouvelles formes d'intégration sociale.

Il revenait à J-P. Bertaud de brosser un tableau des interprétations du coup d'État de Brumaire qui, au cours du xixe siècle, ont fait de Bonaparte alternativement le «sauveur» (avant le coup d'État de son neveu en 1851) puis «l'assassin» (entre les années 1850 et 1880) de la Révolution; cependant, il semble que les lectures du coup d'État de Brumaire, depuis les années 1880, véritables débuts de la Troisième République, oscillent entre l'interprétation héroïque et la légende noire. Enfin, dans sa conclusion, J-P. Jessenne pose des jalons sur la nécessité de prolonger l'ensemble des recherches sur la période consulaire. À ces deux volumes, destinés à être lus en parallèle, s'ajoutera bientôt celui des actes d'une troisième rencontre, qui s'est tenue en mars 2000 à Rouen pour prolonger ces études et ces débats sur la transition du Directoire au Consulat. 